\title{
Visión holista e investigación científica
}

\section{Resumen}

El Holismo como visión generalizadora explica que la realidad es una, las múltiples y heterogéneas manifestaciones no es sino el despliegue del ser esencial. Esta kosmovisión considera que el cosmos o fisiosfera (mundo físico), la bios o biosfera (el mundo de la vida), la psique o nous (el domino de la mente) y la theos o teosfera (domino de lo espiritual) forman una unidad a manera de un solo campo unificado y son un expresión específica de esa totalidad $u$ holismo real. El conocimiento de esta realidad multifacética es posible. Cada nivel tiene sus propias características y requiere de abordajes científicos distintivos, con estrategias y metodologías específicas, esta realidad única, pero de carácter multifacético ha dado lugar al reconocimiento del pluralismo epistemológico, basados en las tres formas correlativas de conocer del ser humano: empírico, racional y espiritual. La epistemología ha resumido los siguientes principios: los tres ojos del conocimiento, los cuatro cuadrantes del ser o el gran tres, el problema de la validez del conocimiento, los errores categoriales, entre las más importantes.
Brígido Ropa Carrión*

\section{Palabras clave:}

holismo, pluralismo epistemológico.

\section{Holistic vision and scientific research}

\begin{abstract}
The Holistic as generalizing vision explains that the reality is one, the multiple and heterogeneous manifestations are the unfolding of being essential. This kosmovisión considers the cosmos or physiosphere (physical world), the bios or biosphere (life world), the psyche or nous (the domain of mind) and Theos or teosfera (the spiritual domain) form a unit by way of a single unified field and they are a specific expression of that whole or real holism. The knowledge of this multifaceted reality is possible. Each level has its own characteristics and requires distinctive scientific approaches, with specific strategies and methodologies, this situation is unique, but multifaceted nature has led to the recognition of epistemological pluralism, based on the three correlative ways of knowing of the being human: empirical, rational and spiritual. The epistemology has summarized the following principles, among the most important: the three eyes of knowledge, the four quadrants of being or the big three, the problem of the validity of knowledge, the categorical errors, etc.
\end{abstract}

\section{Keywords:}

holism, epistemological pluralism. 


\section{Visión holista}

Visión holonómica, visión globalizadora, "una verdadera teoría de campo unificado que engloba la ciencia, la filosofía y la religión".

(Wilber, Ken. 1994, p. 11).

La Visión Holista, denominada también "visión comprehensiva", "visión holonómica" o "visión globalizadora", es una verdadera teoría de campo unificado que engloba los aportes de la ciencia, de la filosofía y de la religión. Como Kosmovisión incluye: cosmos o fisiosfera, la bios o biosfera, la psique o nous o noosfera y la theos o teosfera o el dominio de lo Divino.

La visión holista se caracteriza por una síntesis multidisciplinar que integra las diversas escuelas y disciplinas psicológicas, filosóficas, sociológicas y antropológicas, así como, tradiciones espirituales que abordan el tema sobre el proceso evolutivo del cosmos y la visión antropofilosófica que de ésta se desprende.

La visión como mapa general de orientación en el desarrollo del conocimiento, ha sido objeto de permanente preocupación tanto de los filósofos y los científicos de todos los tiempos. En general, la visión se define “.... como una constelación de creencias, valores y técnicas compartidos por los miembros de una comunidad científica determinada..." (Grof, S. 2001, p. 31) o por las corrientes filosóficas. Así podemos hablar de visiones de ciencias específicas o de las ciencias naturales, sociales y del pensamiento; como también de las variadas corrientes filosóficas como la visión de la Filosofía Perenne, del empirismo, racionalismo, trascendentalismo, etc. Todas ellas poseen una concepción o un sistema específico de pensamiento que sirve de base a las explicaciones de sus respectivas teorías que dan cuenta de las diferentes manifestaciones de la realidad.

Según Wilber, K. (1997, p.39) el Kosmos en el sentido que usaron los pitagóricos es un concepto que incluye no sólo al cosmos, sino también y al proceso pautado de todos los dominios de la existencia. Es decir, "... incluye: COSMOS o fisiosfera, LA BIOS o biosfera, LA PSIQUE o nous o noosfera y LA THEOS o teosfera o el dominio de lo Divino". Por tanto, el kosmos es el sistema integral de toda la realidad y su modelo expresa el paradigma por excelencia que guía el accionar de los hombres, en todos los ámbitos de vida.

La visión Holista explica el curso del desarrollo evolutivo a través de los diversos dominios, desde la materia o fisiosfera, hasta la vida o biosfera, de esta hasta la mente y la razón o nous o noosfera, así como el dominio de lo Divino, theos o teosfera. Abordaje que incluye e integra la materia, el cuerpo, la mente, el alma y el espíritu. Constituye el "Gran nido del ser", porque todo el reino del kosmos está interrelacionado. Este todo, está ocurriendo, siendo o evolucionando continua y permanentemente y sin descanso alguno, por así decirlo. Muestra al universo como una trama unificada e indivisible de sucesos y relaciones, cuyas partes integrantes representan distintos aspectos y pautas de un único proceso integral de inimaginable complejidad. El principio de conexión de la trama kósmica es la consciencia, atributo primario e irreducible de la existencia. Como todo está estrechamente interrelacionado, el hombre no es excepción, podemos experimentar esta unidad holística con el kosmos. Siguiendo esta línea de pensamiento holístico, el filósofo Jan Smults decía (Citado por Wilber 2002, p. 14): 
Miremos hacia donde miremos, sólo veremos totalidades. Y no sólo simples totalidades, sino totalidades jerárquicas; cada totalidad forma parte de una totalidad mayor que, a su vez, está contenida dentro de otra totalidad aún más inclusiva. Campos dentro de campos que se hallan dentro de otros campos, campos que se extienden a lo largo de todo el cosmos, interrelacionando así todas y cada una de las cosas.

La visión Holista nos muestra un mapa general en el que todo está interrelacionado o íntervinculado, donde no hay nada, como manifestación de la realidad, que esté separado del todo. Este concepto del todo vinculado es la expresión concreta de un sistema, y los pensadores de nuestros días coinciden en conceptuar "...como un conjunto de elementos relacionados entre sí y armónicamente conjugados..." (Ferrater, J. 1995, p. 687).

La holística, al considerar el proceso del desarrollo del Kosmos como un sistema complejo y permanente, sujeta a principios que norman el devenir continuo de la realidad. Entre los principios más importantes tenemos los siguientes:

A. La realidad está compuesta de totalidad/parte u holones: Por ejemplo la célula forma parte de los tejidos biológicos y estas forman parte de órganos, por tanto, la célula es una totalidad, pero también es parte de una totalidad mayor como son los tejidos biológicos. El mundo está compuesto de holones, las partículas subatómicas no son sino holones.

B. Los holones son regidas por cuatro impulsos: Individualidad, totalidad, auto trascendencia y autodestrucción: Todos los holones, en los diferentes niveles, participan de la tendencia de mantener su propia individualidad, identidad, su propia autonomía, su propia actividad, si fracasan desaparecen. Pero, los holones también gozan la tendencia a formar parte de algún otro sistema o alguna otra totalidad mayor. Lo fascinante es que todos los holones tienden al impulso de auto trascendencia o el impulso a ascender a un nivel superior; un impulso que tiene la asombrosa capacidad de ir más allá de donde se encontraba anteriormente. Los holones también poseen el impulso a descender a un nivel inferior, llamada impulso de autodisolución o disgregación en los sub holones que lo componen.

C. Los holones emergen holoárquicamente: significa un orden de totalidad creciente, una "jerarquía" anidada. La evolución tiene lugar o discurre jerárquicamente; es decir, discurre por medio de holoarquías naturales hacia los órdenes de holismo y totalidad creciente. La única alternativa realmente holista es la holoárquica. Nótese bien, esta holoarquía es una jerarquía natural: Por ejemplo los valores pre convencionales son ampliamente superados por los valores convencionales y estas por los valores mundicéntricos.

D. Cada holón emergente trasciende pero incluye a su(s) predecesor (eres): Los holones son totalidad/parte, la totalidad trasciende, pero las partes son incluidas, aceptadas, integradas y unidas a la totalidad. La evolución es un proceso de trascendencia e inclusión. Una molécula trasciende el átomo, en la primera aparecen nuevas propiedades emergentes e incluye los átomos. Los átomos son contenidos en las moléculas y no viceversa. Este no viceversa 
determina la holoarquía o el orden de la totalidad creciente, niveles de organización estructural. Las totalidades dependen de las partes, pero no viceversa. La biosfera trasciende e incluye la fiosfera, así como la noosfera trasciende e incluye la biosfera. En la evolución del hombre: El cuerpo es trascendido e incluido por mente, esta es trascendido e incluido por el espíritu. Pero, qué pasaría si se niega y se rechaza al cuerpo, terminará reprimido.

E. La evolución procede creando niveles sucesivamente más profundos y menos amplios: La evolución produce más profundidad y menos amplitud. Existen menos organismos que células, menos células que moléculas, menos moléculas que átomos y menos átomos que quarks. Cada uno de esos niveles tiene una mayor profundidad pero también menor amplitud.

F. La evolución tiende hacia una complejidad creciente: A largo plazo, la evolución sigue un telos, una dirección. El impulso básico de la evolución es la profundidad creciente, este es el impulso de auto trascendencia del kosmos, de ir más allá en donde estaba anteriormente, incluyendo lo que era y aumentando su grado de profundidad. El hombre forma parte de este proceso evolutivo. La evolución tiende, de manera general, a moverse en la dirección de una complejidad creciente, de una diferenciación/integración creciente, de una organización y estructuración creciente, de una autonomía relativa creciente, de un telos creciente. Pero, también le es inherente a la evolución la regresión y la disolución. Estas dos tendencias se presentan a largo plazo.

La Filosofía Perenne comparte esta visión: La Realidad, el Kosmos es una Gran holoarquía anidada de ser y de conciencia, que va de la materia, a la vida, la mente y el espíritu. Cada dimensión trasciende e incluye a las dimensiones inferiores que se representan mediante círculos concéntricos de sistemas cada vez más crecientes y profundos. Por tanto podemos postular que la realidad no es sino un conjunto de sistemas dentro de otros sistemas más profundos y de creciente complejidad, cuyo conocimiento engrandecerá al ser humano y en un escenario no muy lejano le permitirá desplegar todo su potencial de dicha y amor incondicional o de plena realización.

\section{La gran cadena del ser y el conocimiento}

En la visión holista:

La realidad consiste en una serie de nidos dentro de nidos que se hallan, a su vez, dentro de otros nidos... con el resultado de que todos los seres y todos los niveles se hallan, en última instancia, englobados en el amoroso abrazo del espíritu omnipotente. (Wilber, K. 1998, p. 21).

Cada nivel superior del Gran Nido del Ser presenta los rasgos esenciales de los niveles inferiores, pero le agrega cualidades distintas, es decir, los trasciende. Por eso, cada nivel de la realidad presenta una configuración o una arquitectura diferente. Esta característica de la realidad ha determinado que las grandes tradiciones de la sabiduría o la filosofía perenne postulen, que cada nivel de la realidad dispone de una rama concreta del conocimiento. Por eso, la ciencia, la filosofía y el misticismo ocupan un lugar en este Gran Nido del Ser. El conocimiento de cada nivel implica abordajes distintivos, con 
estrategias y metodologías específicas. Esta visión trazado por el holismo ha dado lugar al reconocimiento del pluralismo epistemológico.

Según Wilber, K. (1998, p. 32) la visión del pluralismo epistemológico ha sido la columna vertebral de las grandes tradiciones de sabiduría y se quebró con el colapso de la Gran Cadena de la que dependía en la modernidad, tendencia que continua hasta la actualidad. Sin embargo, para los pocos eruditos, teóricos y legos inteligentes que tratan de buscar el sentido del universo en algún abordaje holístico o global, el pluralismo epistemológico constituye la base para la visión científica de la actualidad.

El pluralismo epistemológico fue reconocido brillantemente por místicos cristianos como San Buenaventura y Hugo de San Víctor cuando decían que cada ser humano dispone de un ojo de la carne, un ojo de la mente, y un ojo de la contemplación. Tres modalidades del conocimiento relacionados con las tres dimensiones correlativas del ser (materia, sutil y causal) y que cada una de ellas es completamente válida e importante, cuando se ocupan de su propio dominio. Es así como disponemos de una visión equilibrada del conocimiento empírico (la ciencia), el conocimiento racional (la lógica y las matemáticas) y el conocimiento espiritual (la gnosis).

\section{La investigación científica}

“...Solo hay un mundo -este imponente y misterioso como lo llama Castañeda, C.- pero esta única realidad tiene múltiples aspectos, múltiples dimensiones y niveles. Los físicos y los místicos se ocupan de aspectos distintos de la realidad..." (Capra, F. 1995, p. 320).

$\mathrm{Si}$, investigar significa averiguar, indagar y buscar, entonces la investigación científica es un proceso que busca la producción y comprobación del conocimiento mediante la aplicación del método científico. Según Romero y Galicia (citado por Díaz, M. et al, 2013, p.15) la investigación científica es la "búsqueda sistemática, reflexiva y metódica, interesado en obtener nuevos conocimientos y solucionar problemas de tipo científico, filosófico, empírico y técnico a través de la observación, reflexión y la experimentación". La visión holista como generalización orientadora, integral y comprensiva ha desarrollado un conjunto de principios epistemológicos aplicables a la investigación científica, tales como: los tres ojos del conocimiento, los cuatro cuadrantes del ser o el gran tres, el problema de la validez del conocimiento, los errores categoriales, entre las más importantes.

\section{Los tres ojos del conocimiento}

Siguiendo la tradición de Hugo San Víctor, San Buenaventura se considera que los hombres poseen tres formas de adquirir conocimientos o tres ojos (Wilber, K. 1994, p. 13):

A. El ojo de la carne, mediante el cual percibimos el mundo externo o material, del espacio, el tiempo y los objetos. El dominio de lo grosero, reino de la comprensión empírica, el mundo de la experiencia sensorial. Es el ojo empírico o de la experiencia sensible que incluye todo aquello que puede ser captado o detectado por los cinco sentidos y sus extensiones. Este ojo de la carne es molológuico, término derivado de "monólogo". La mayor parte de las ciencias empíricas son 
monológuicas, ya que la investigación se realiza sin habla con el objeto de estudio.

B. El ojo de la razón que nos permite alcanzar el conocimiento de la Filosofía, de la Lógica y de la mente, reino de meditatio y de las verdades psíquicas. El ojo de la mente participa del mundo de las ideas y del pensamiento, de las imágenes, de la lógica y de los conceptos. A la luz del intelecto podemos ver cosas invisibles para nuestros sentidos corporales. La verdad de una deducción lógica no depende de su relación con los objetos sensoriales sino de su consistencia interna. El ojo de la razón es dialológuico, que procede de "diálogo" lo que significa hablar con alguien e intentar comprenderlo.

C. El ojo de la contemplación, mediante el cual tenemos acceso a lo trascendente. Reino de la contemplación, donde el psiquismo o el alma se unifican con la divinidad en la intuición trascendente. La contemplación trasciende la razón. Este ojo es transracional, translógico y trascendental. La contemplación trasciende e incluye la razón e implica la contemplación de lo inmutable de la identidad real.

En general, todos los hombres y las mujeres poseen un ojo carnal, un ojo racional y un ojo contemplativo, cada uno tiene sus propios objetos de conocimiento; un ojo superior no puede ser reducido a un ojo inferior ni explicado por él; que cada ojo es válido y útil en su propio dominio pero incurre en una falacia cuando intenta captar totalmente los ámbitos superiores o inferiores.

\section{Los cuatros cuadrantes}

La Gran Cadena del Ser constituye una jerarquía en la que cada nivel superior trasciende, pero incluye, a sus predecesores. La mejor forma de representarla es mediante una serie de círculos concéntricos, de nidos, donde cada nido superior envuelve o engloba a sus predecesores. Estas jerarquías presentan cuatro "rostros" del kosmos que son la trama y la urdiembre del tejido de lo real y del cual la ciencia, la filosofía y la sabiduría del oriente dan cuenta en forma reiterada en los diferentes periodos históricos.

Estos cuatro tipos de jerarquías, llamadas también cuatro cuadrantes, están relacionadas con el interior y el exterior de lo individual y de lo colectivo de la realidad. Cada uno son diferentes, pero se hallan profundamente interrelacionadas e interconectadas de un modo intrínsecamente necesario. Estas jerarquías son la base para establecer diferentes tipos de conocimiento y definir la naturaleza del conocimiento científico. En la siguiente grafica observamos los cuatro cuadrantes.

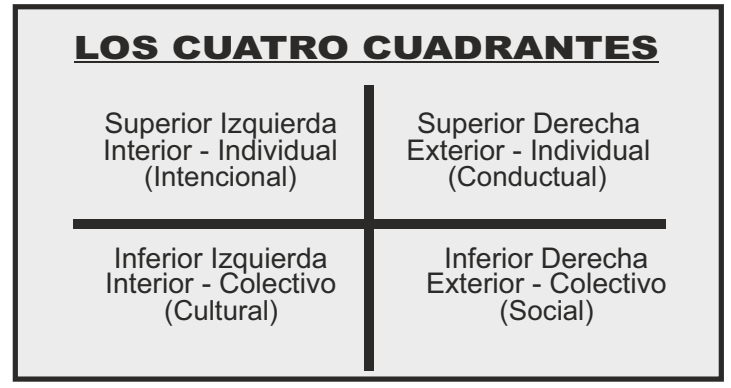

Fuente: elaboración propia a partir de los conceptos de Wilber, $K$. 
El cuadrante superior derecho o exterior del individuo. Cuadrante del relato científico estándar de los componentes individuales del universo: átomos, moléculas, células individuales, organismos multicelulares y los organismos superiores provistos de cuerda neural, tronco cerebral reptiliano, sistema límbico, neocortex y neocortex complejo. (Wilber, K. 1998, p.89).

El cuadrante inferior derecho (llamado también lo social), representa las comunidades o sociedades de esos holones, como son las galaxias, la tierra, la sociedad desde las más primitivas hasta la comunidades más desarrolladas que conoce el hombre. Este cuadrante es simplemente un resumen que nos brinda la ciencia empírica y sistémica de la forma en que evolucionan las modalidades colectivas de los holones.

En el cuadrante superior izquierdo observamos la holoarquía de la conciencia interior. Esta va desde la simple aprehensión a la irritabilidad, la sensación, la percepción, el impulso, la emoción, las imágenes y los símbolos, los conceptos, las reglas y las operaciones concretas, la cognición reflexivo-formal y la visión creativa (Visón-lógica). Representa la conciencia individual, interiory subjetiva.

El cuadrante inferior izquierdo representa las formas de conciencia colectiva o intersubjetivas, los significados, los valores y los contextos culturales compartidos; sin los cuales la conciencia individual no se desarrolla ni funciona en modo alguno. Cuando las cogniciones individuales y subjetivas son compartidas con otros individuos, el resultado es una visión del mundo o una perspectiva colectivamente compartida.

Los dos cuadrantes de la mano derecha representan las realidades objetivas o exteriores y que los dos cuadrantes de la mano izquierda representan las realidades subjetivas o interiores. En la investigación objetiva, empírica, científica, los holones vistos desde el exterior, constituye la realidad de la mano derecha; mientras los cuadrantes de la mano izquierda, son los holones contemplados desde el interior, como parte de la conciencia y de la experiencia inmediata.

Todo lo que se halla en la mano derecha tiene localización simple, localización en el mundo empírico y sensorio motor; pero lo que se halla en la mano izquierda carece de localización simple porque los holones no se encuentran en el espacio físico sino en el espacio emocional, mental y en el espacio cognitivo, por tanto son espacios intencionales.

Además, los cuatro cuadrantes del cosmos se relaciona con los tres dominios descubiertos por la modernidad: yo, nosotros y ello o arte, moral y la ciencia. El superior izquierdo se describe en el lenguaje del "yo", el cuadrante inferior izquierdo se describe en el lenguaje de "nosotros" y los dos cuadrantes de la mano derecha, al ser exterioridades objetivas, se describe en el lenguaje de "ello". Por tanto, también está relacionado con los conceptos de bello, lo bueno y lo verdadero.

Yo, ubicado en el superior izquierdo del cuadrante: conciencia, subjetividad, el yo como expresión de uno mismo que incluye el arte y la estética; la conciencia vivida irreductible e inmediata; el relato en primera persona.

Nosotros (inferior izquierdo): la ética, la moral, las visiones del mundo, los contextos comunes, la cultura; el significado intersubjetivo, la comprensión mutua, la justicia, el relato en la segunda persona plural.

Ello, mano derecha: La ciencia, tecnología, la naturaleza objetiva, las formas empíricas, incluyendo el cerebro y el sistema social; la verdad proposicional, exterioridades 
objetivas de los individuos y los sistemas; el relato en tercera persona.

El gran tres es reconocido por un gran número de eruditos. Karl Popper habla de los subjetivo (yo), cultural (nosotros) y objetivo (ello). Las tres pruebas de Habermas: la sinceridad subjetiva (yo), el ajuste intersubjetivo (nosotros) y la verdad objetiva (ello). Se evidencia también en la decisiva trilogía de Kant: La crítica de la razón pura (la ciencia objetiva), La crítica de la razón práctica (la moral) y La crítica del juicio estético (el arte), (Wilber, k. 1998, p. 96). Así como, las más variadas tradiciones de los místicos del oriente y la visión del mundo de la civilización andina: el mundo de arriba, el mundo de aquí y el mundo de abajo.

\section{El problema de la validez del conocimiento}

Una de las cuestiones fundamentales de la Epistemología, en su sentido estricto, es el problema de la validez de los conocimientos y en esta parte, Ken Wilber plantea que la gran pregunta a responder es: ¿Cómo podemos estar seguro de la validez del conocimiento obtenido por cualquiera de las tres ojos o vías señaladas anteriormente? o ¿Qué es lo puede permitirnos refutar la validez de un determinado conocimiento?

Los datos constituyen la aprehensión directa de la experiencia, entendida como captación o conciencia. Los ámbitos de la sensibilia (carne), intelligibilia (mente) y del espíritu (trascendelia) nos ofrecen datos legítimos y reales posibles de ser aprehendidos. La existencia de esos dominios objetales y la realidad de sus datos constituyen el fundamento de la búsqueda del conocimiento y la mejor garantía de su posible consecución.

Sea cual fuere el ámbito que consideremos, la característica distintiva de los datos está en el hecho de manifestarse inmediatamente ante nuestra conciencia como una aprehensión directa, como algo dado.

La aprehensión inmediata no es igual en lo sensorial, en lo mental y en intuición espiritual. Las experiencias directas o intuiciones de la realidad material o sensibilia, como el caso del libro, una flor son experiencias sencillas; pero también pueden ser muy complejas como una puesta del sol, experiencias como las de un fotón, el firmamento nocturno, etc.

Los datos propios de la intelligibilia como nuestra experiencia mental presente dada e inmediata como una imagen o un pensamiento fugaz que son muy simples, pero también puede ser complejo y permanente como un recuerdo de una frase de una idea. Esta percepción es el dato, trátese de una palabra, de una frase o de una idea, el dato mental es nuestra experiencia mental inmediata.

En el ámbito de trascendelia, el dato trascendental percibido o intuido directamente e inmediatamente por el ojo de la contemplación, puede ser una intuición espiritual, una iluminación, intuición gnóstica, un satori.

En los círculos académicos existe mucha controversia acerca del significado del término experiencia, toda vez que los empiristas han insistido siempre en que todo conocimiento debe basarse en la experiencia. El núcleo del problema es la gran ambigüedad del significado de la palabra "experiencia".

Los empiristas reducen el significado de la palabra experiencia a lo sensorial. Sin embargo, la experiencia también es posible en los ámbitos de lo mental y espiritual: Todos nosotros experimentamos sensaciones y percepciones; el ojo de la mente puede 
experimentar el discurrir de un pensamiento, de las ideas y de la imaginación; del mismo modo podemos experimentar el espíritu por medio del ojo de la contemplación o gnosis y ser testigos de la experiencia de la iluminación, anata, la unicidad del ser.

El término experiencia es sinónimo de percepción directa, de lo inmediatamente dado, de la intuición en los tres ámbitos del conocimiento. Todo conocimiento está basado en la experiencia (como afirman los empiristas), pero no necesariamente en la experiencia sensorial. Es indudable la existencia de modalidades del conocimiento que aunque permanezcan al margen o fuera de las experiencias sensoriales y no por ello dejan de ser experiencias.

Los empiristas afirman que todo conocimiento real es experimental, lo cual es cierto; pero, inmediatamente agregan que no existe más que la experiencia sensible o sensorial; esto, es indudablemente falso y constituye la esencia del reduccionismo del empirismo.

Para verificar los datos se usan los principios de verificación o refutación de datos que se aplica a la recogida de datos en cada uno de las modalidades del conocimiento. Al abordar estos procedimientos de recogida y verificación de datos es muy importante tener en cuenta dos puntos esenciales:

A. La metodología real de recogida y de verificación de datos son completamente diferentes en cada una de las modalidades del conocimiento.

B. Los principios abstractos de recogida y verificación de datos son esencialmente idénticos.

Los principios abstractos de recogida y verificación de datos en cualquiera de los ámbitos del conocimiento son tridimensionales:

1. Prescripción instrumental: Tiene siempre la forma "si quieres saber esto deberás hacer este otro". Se trata de una práctica real.

2. Aprehensión intuitiva: Consiste en la percepción cognitiva, aprehensión o experiencia directa e inmediata de los datos, del ámbito objetal o conocimiento. Se trata de experimentar el dominio definido por el paso anterior.

3. Confirmación comunal: Consiste en el cotejo de los resultados obtenidos con otras personas que también hayan pasado por los estadios perceptivos o preceptivos.

Cada una de las tres modalidades del conocimiento, lo sensorial, mental y espiritual, nos permite acceder a un ámbito diferente de aprehensiones, o datos directos, inmediatos e intuitivos. Pero, los datos propios de la modalidad mental (palabras, símbolos y conceptos) son simbólicos, intencionales, reflexivos y referenciales, y pueden ser utilizados para señalar o representar, los datos procedentes de otros dominios: sensibilia, la misma intelligibilia y trascendelia.

\section{Conclusiones}

La Visión Holista, Comprehensiva u Holonómica da cuenta de la totalidad del despliegue de la realidad y en esta perspectiva establece la totalidad de las niveles, tipos y modalidades del conocimiento; sin desdeñar, ni condenar y con la objetividad sin 
parangón, demuestra la existencia de los tres niveles del conocimiento: El conocimiento empírico-analítica, el conocimiento racional o simbólico-lógico y el conocimiento trascendental o transracional, transverbal.

Desde el punto de vista de la Epistemología, cada nivel del conocimiento tiene su propio objeto de estudio, sus propias estrategias metodológicas, procedimientos, técnicas e instrumentos de investigación y principios abstractos iguales para demostrar la validez de sus respectas verdades. Estos principios abstractos válidos son: A). El aspecto instrumental o perceptivo del tipo "si haces esto, entonces lograrás lo siguiente...". B). Un aspecto aprehensivo o visión iluminativa o luz interior para cada ojo particular. C). Un aspecto comunal, cuyos reputados miembros confirman o desmienten el cospus teórico y de los logros de los conocimientos y de las verdades.

Es indudable la existencia de los reinos de la sensibilia, intelligibilia y trascendelia, por la abundante información que dan cuentan las disciplinas científicas y en especial de la trascendelia por los trascendentales logros alcanzados por la Psicología transpersonal, la Antropología, las escuelas místicas del oriente, de los gnósticos y de las paraciencias.

\section{Referencias Bibliográficas}

Capra, C. (1995) El Tao de la Física. México: Grijalbo.

Díaz, M.; Escalona, M.; Castro, D.; León, A. y Ramírez, M. (2013). Metodología de la investigación. México: Trillas.

Ferrater, J. (1995) Diccionario Filosófico. Buenos Aires: Editorial Sudamericana.

Grof, S. (2001) Psicología Transpersonal. Barcelona: Editorial Kairós.

Wilber, K. (2002) Proyecto Atman. Barcelona: Editorial kairós.

---. (1998) Ciencia y Religión. Barcelona: Editorial Kairós.

---. (1997) Breve Historia de Todas las Cosas. Barcelona: Editorial Kairós.

---. (1994) Los Tres Ojos del Conocimiento. Barcelona: Editorial Kairós. 